\title{
Alveolar Ridge Preservation in Mandibular Molars Using Mixture of Anorganic Bovine Bone (ABB) and Autogenous Particulate Vs Mixture of Injectable Platelets Rich Fibrin, ABB and Autogenous Particulates (Sticky Bone) (A Randomized Clinical Trial)
}

\author{
Mohamed Darwish Elsayed Darwish ${ }^{1 *}$, Nevien Abd El-Latif Askar², \\ Mohamed Atef Abdel-Rasoul ${ }^{2}$ and Hatem Abdel-Fattah Amer ${ }^{3}$ \\ ${ }^{1}$ Department of Oral Implantology, Faculty of Dentistry, Cairo University, Egypt \\ ${ }^{2}$ Department of Oral and Maxillofacial Surgery, Faculty of Dentistry, Cairo University, \\ Egypt \\ ${ }^{3}$ Department of Oral and Maxillofacial Pathology, Faculty of Dentistry, Cairo \\ University, Egypt \\ *Corresponding Author: Mohamed Darwish Elsayed, Department of Oral \\ Implantology, Faculty of Dentistry, Cairo University, Egypt.
}

Received: December 21, 2020

Published: January 22, 2021

(C) All rights are reserved by Mohamed

Darwish Elsayed Darwish., et al.

\section{Abstract}

The aim of the study was a Radiographic and Histological assessment using a Mixture of Anorganic Bovine Bone (ABB) And Autogenous Particulate bone vs a Mixture of Injectable Platelets Rich Fibers, ABB and Autogenous Particulates bone (sticky bone) In socket preservation. The study question was "does socket preservation of the Alveolar Ridge in the lower posterior regions yield better quality and quantity of bone using a Mixture of Anorganic Bovine Bone(ABB) And Autogenous Particulate bone or a Mixture of Injectable Platelets Rich Fibrin, ABB And Autogenous Particulates bones (sticky bone)?".

Methodology: Radiologic assessment using CBCT after extraction and socket preservation and CBCT before dental implants as well as histologic assessment of core biopsy sample.

Result: Sticky bone showed a statistically significant higher mean value of bone area percent compared to a mixture of ABB and Autogenous bone. Sticky bone is better than the mixture of ABB and Autogenous particulate bone in regards to the amount of bone formation. .

Conclusion: The results are increased predictability in preserving the socket from collapse which in turn will result in a more successful prosthetically driven dental implant placement surgery.

Keywords: Socket Preservation; Bone Resorption; Sticky Bone; Extraction; Dental Implants

\section{Introduction}

Healing after tooth extraction is always accompanied by ridge resorption. Resorption occurs mostly during the early healing period (from 3 to 12 months). Some studies have attempted primary wound closure using the similar concept of guided bone regeneration [1].

The challenge of preserving a socket is amplified when presented with ridge defects, as usually a clinician is faced with many problems in soft and hard tissue regeneration [2].
A few different donor sites from approximate or distant regions of membranous or endochondral bone are available however autogenous bone grafts have the disadvantages of donor site morbidity and the limited amount of available graft material. To overcome the disadvantages of autogenous bone grafting, the use xenogeneic bone grafts for bone regeneration has been utilized [3].

Alveolar ridge resorption has an obvious impact on implant placement, especially when implant-supported restorations are planned as a therapy (Seibert and Salama, 1996 [4]). That's why, 
socket preservation after extraction (ARP) has become a key component of clinical dentistry.

\section{Bone graft mechanism}

Bone grafting may have one or more biological mechanism that provide a rationale for its use. The mechanisms are: osteoconduction, osteoinduction, and osteogenesis [5]

\section{Osteoconduction}

This mechanism happens when the graft material acts as a scaffold for growth of new bone, which is enhanced by the natural bone. Osteoblasts from the defect that is being grafted, use the graft material as a framework to spread new bone. [6] Every graft material has to be osteoconductive.

\section{Osteoinduction}

Osteoblasts are differentiated from the stimulation of osteoprogenitor cells and then begin formation of new bone [5].

\section{Osteopromotion}

Enhances the osteoinduction mechanism without affecting its properties [5].

\section{Osteogenesis}

Occurs when osteoblasts originating from graft material contribute to the growth of new bone along with bone formation [7]

\section{Socket preservation}

The first attempts to decrease bone resorption were performed by a root shield technique (Osburn, 1974). Nevertheless, root retention is not always feasible because of tooth decay and/or fractures amongst other reasons. Alveolar ridge preservation using socket grafting technique took place in the mid-1980s as an alternative to the root shield technique. It is based on the idea that filling the socket with a biomaterial would stimulate a "root retention effect" [8]. This approach showed continued success for decades.

It is possible to preserve the height and width of the ridge by using socket preservation techniques. Atraumatic tooth extraction is the first step for socket preservation. Socket preservation can be done by placement of bone graft material, membrane (e.g. collagen membrane), and/or a connective tissue graft $[9,10]$.
Bone graft

Autogenous bone

AAutogenous bone grafting is the reposition of the patient's own bone from one site to another. Autografts are biocompatible and have the ability to form new bone through osteogenesis, osteoinduction, and osteoconduction (AlGhamdi., et al. 2010 [11]). Autogenous grafts are cancellous, cortical, or cortico-cancellous. Cancellous bone is preferred because it is rapidly re-vascularized and is seldom rejected by the graft site (Porrini., et al. 2011 [12]).

(Johansson., et al. 2001 [13]) used computed tomography (CT) analysis to evaluate the autogenous bone resorption in patients with severely atrophic edentulous maxilla treated with onlay grafts and particulate bone grafts for maxillary sinus lifting. CT scans were obtained in the first two weeks postoperatively and after 6-7 months. In this time, the volumes of the inlay and onlay grafts were reduced on average by $49.5 \%$ and $47.0 \%$ of the initial volumes respectively.

\section{Allografts bone}

The allografts are obtained from other individuals of the same species but disparate genotype. They include freeze-dried bone allografts (FDBA) and demineralized freeze-dried bone allograft (DFDBA). Bone allograft are the most frequently used alternative to autogenous bone for bone grafting procedures in the USA (Reynolds., et al. 2010 [14]).

To avoid disease transmission from allografts, several chemical and physical processing techniques have been used. Chemical treatment with agents, such as $5 \%$ peracetic acid, $0.1 \%$ ethylene diamine tetra-acetic acid, or $0.1 \%$ sodium dodecyl-sulfate, can alter the bone structure but may not sufficiently inactivate pathogens. Physical treatment, such as ultrasonication, may alter the microcrystal structure of bone mineral and denature organic components. With FDBA and DFDBA, more satisfactory results have been obtained through lyophilization, but cellular debris might remain after this treatment that could interfere with healing (AlGhamdi., et al. 2010 [11]).

\section{Alloplast bone}

An alloplastic material is a biocompatible, inorganic synthetic bone grafting material. At present, alloplasts marketed for periodontal regeneration fall into two broad classes: ceramics and polymers. The fate of an alloplastic bone grafting material is depen- 
Alveolar Ridge Preservation in Mandibular Molars Using Mixture of Anorganic Bovine Bone (ABB) AND Autogenous Particulate vs Mixture of Injectable Platelets Rich Fibrin, ABB and Autogenous Particulates (Sticky Bone) (Randomized Clinical Trial)

dent primarily on its chemical composition, structure and physical properties (Reynolds., et al. 2010 [14]).

\section{Xenografts}

Xenografts can be taken from many sources, including (coralline, bovine, equine, and porcine), they are generally biocompatible and similar to human bone in structure. Xenografts are osteoconductive but are not osteoinductive in humans and less frequently associated with the formation of interposition areas of connective tissue (AlGhamd., et al. 2010 [11]).

Synthetic biomaterials are better when compared to the animal biomaterials, regarding to the higher risk of inflammatory reactions and disease transmission caused by the animal biomaterials (Rodella., et al. 2011 [15]).

Bovine xenografts may contain similar hydroxyapatite content as that exist in the of human bone, that allows the graft to rapidly revascularize and be replaced by new bone (AlGhamdi., et al. 2010 [11]).

\section{Growth factors}

Growth factors play an important role in repairing or generating damaged tissue. Platelets release high quantities of growth factors [16].

Many techniques to collect platelet aggregate have been attempted to accelerate tissue healing in medical field and in dental field as well [17].

\section{Platelet rich plasma and platelet rich growth factor}

(Marx., et al.) was the first to introduce Platelet rich plasma as a platelet aggregate. It has been widely used in the dental field especially in advanced surgical procedures such as: ridge augmentation, sinus augmentation, periodontal regeneration and soft tissue healing. PRP and PRGF extract platelet concentrates use pipetting after centrifugation of the extracted blood in the centrifuge. The procedure shows some errors to form proper platelet concentrates. They use only $10 \%$ of the venous blood. PRF and CGF overcome these disadvantages of PRP and PRGF.; PRF and CGF do not need any biochemical additives such as calcium chloride or bovine thrombin, to make gel condition. That is why they are free from risk of cross contamination [18].
Platelet rich fibrin

Platelet rich fibrin (PRF) is a fibrin matrix in which platelet cytokines, growth factors, and cells are trapped and may be released after a certain time and that can serve as a resorbable membrane. Choukroun [19] and his associates were amongst the pioneers for using PRF protocol in oral and maxillofacial surgery to improve bone healing in implant dentistry. Autologous PRF is a healing biomaterial, and presently, studies have shown its application in various disciplines of dentistry. .

\section{Concentrated growth factor}

It was introduced by (Sacco in 2006). It is a better version of PRF with a strengthened fibrin matrix and boosted growth factors and cytokines. CGF technology has a very good characteristicsuch as the easy and speedy one-step preparation of larger, denser and richer growth factors fibrin matrix than the other solid PRPs. There is a presence of fibrin network constituted by thin and thick fibrillar elements with multiple elements trapped among the fibrin network [20].

CGF seems to possess a good regenerative capacity and versatility. For example, it has been reported that CGF has a positive effect on: sinus and alveolar ridge augmentation [6].

Guided bone regeneration (GBR) using bone graft and barrier membrane is a well established technique for augmentation of alveolar ridges [21,22]. For successful GBR; stability of bone graft, space maintenance, angiogenesis and tension free primary closure are essential [22].

Sticky bone

Sticky bone is defined as autologous fibrin glue (AFG), it is prepared by taking 20-60CC of patient's venous blood, the blood is divided to one to two non-coated vacutainers to obtain autologous fibrin glue (AFG), which will make sticky bone. The centrifugation time for AFG varies from 2-12 minutes, Space maintenance with particulate bone graft should be provided during healing period. However, particulate bone graft easily migrates when grafted on the large horizontal/vertical bone defect. To reconstruct large wall bony defect, collagen membrane or titanium mesh is required to 
Alveolar Ridge Preservation in Mandibular Molars Using Mixture of Anorganic Bovine Bone (ABB) AND Autogenous Particulate vs Mixture of Injectable Platelets Rich Fibrin, ABB and Autogenous Particulates (Sticky Bone) (Randomized Clinical Trial)

contain particulate bone graft during healing but these procedures are surgically time consuming and technique sensitive. As an alternative to titanium mesh or block bone procedure, sticky bone was introduced in 2010. It is basically a solidified bone graft which is entrapped in fibrin network. It does not scatter even upon being shaken with cotton pliers because particulate bone powders are strongly interconnected with each other by a fibrin network. These platelet concentrates have shown promising results and have been developed with an idea to combine the fibrin sealant properties with the growth factors in platelets thereby providing an ideal base for wound healing and regeneration of tissues [23].

\section{Research objective}

Radiographic and Histological assessment using a Mixture of Anorganic Bovine Bone (ABB) And Autogenous Particulate vs a Mixture of Injectable Platelets Rich Fibrins, ABB And Autogenous Particulates (sticky bone) In socket preservation.

\section{Materials and Methods}

Study was conducted in the clinic of Masters program for oral implantology (out-patient), Faculty of Dentistry, Cairo University, Egypt. Twenty candidates (of both genders) suffering from nonrestorable mandibular molars and seeking implant rehabilitation were selected. This study was approved by the ethics committee the Faculty of Dentistry, Cairo University.

\section{Eligibility criteria}

\section{Inclusion criteria}

- $\quad$ Adult patients with non-restorable mandibular molars indicated for extraction and implant placement in two stages that indicate socket preservation.

- 18 to 60 years old patients

- Both genders.

- No intraoral soft or hard tissue pathology.

- No systemic conditions that contraindicate implant placement (ex: uncontrolled diabetes mellitus or osteoporosis).

\section{Exclusion criteria}

- $\quad$ Presence of fenestrations or dehiscence of the residual bony walls after extraction.

- Patients with systemic disease that may affect normal healing.

- $\quad$ Psychiatric problems.

- $\quad$ History of radiation therapy to the head and neck.

- $\quad$ Patients that refuse to be called back for implant placement post extraction.

General characteristics for both groups

Once enrollment was done and written informed consent was obtained, eligible patient were randomized in equal proportions prior to initiation of treatment to prevent bias that may have occurred if randomization had occurred after tooth extraction. Patients were randomized into groups between study group (socket preservation using a mixture of Injectable Platelets Rich Fibers, ABB And Autogenous Particulates (sticky bone) and a control group where socket preservation post-extraction was carried out using a mixture of Anorganic Bovine Bone (ABB) and Autogenous Particulate).

First stage: extraction and socket preservation

Patients of both groups are subjected to:

- An intraoral and extraoral diagnosis. Medical and dental history was taken, all the information was written in a diagnostic chart.

- An informed consent was signed by the patient after reading and understanding all the points included in the consent (in the Arabic language, the mother tongue for the Egyptian patients).

- Standard panoramic radiograph before extraction (Figure 1).

- $\quad$ Primary impressions and intraoral photographs were obtained (Figure 2).

- Inferior alveolar nerve block and long buccal nerve block local anesthesia was given to the patient ( $2 \%$ sedicaine with 100,000 epinephrine). In case of autogenous bone particulate harvesting from the chin area, incisive nerve block was applied. 
- A peritome was used for atraumatic extraction of roots.

- Socket debridement using a bone curette was applied after extraction.

- $1 \mathrm{~cm}$ incision will be made in the chin area.

- Neo Biotech ACM Auto Chip Maker 4.5mm diameter, $14 \mathrm{~mm}$ length was used to harvest the autogenous bone from the chin (Figure 6).

- The harvested autogenous bone was collected from Neo Biotech ACM Auto Chip Maker 4.5mm diameter $14 \mathrm{~mm}$ length (Figure 10).

- Xenograft was prepared in a 50:50 ratio to the collected $\mathrm{Au}$ togenous bone (Figure 8).

- $\quad$ Suturing of the chin using non-resorbable polypropylene suture (continuous with lock).

In the study group

The socket will be fully packed with a mixture of Injectable Platelets Rich Fibers, ABB And Autogenous Particulates (sticky bone) (Figure 9).

\section{Sticky bone preparation}

- $20-60 \mathrm{cc}$ of patients' venous blood is drawn from the forearm.

- Blood is rapidly injected to one non-coated test tube to obtain autologous fibrin glue (AFG), the tube insert a slot in the centrifuge, The opposite slot of the centrifuge is filled with a water-filled test tube for weight balance.

- The blood in the test tubes was centrifuged at 2400-2700 rpm using specific centrifuge with a rotor turning at alternated and controlled speed for 12 minutes. The centrifugation time for AFG varies from 3-5 minutes. To get higher growth factors, the centrifuge is stopped after 2 minute-centrifugation and take AFG tube out of the centrifuge.

- $\quad$ The non-coated tube shows 2 different layers. The upper layer is an autologous fibrin glue (AFG) layer and the red blood cells sediment in bottom layer which was discarded.
- $\quad$ The upper AFG is obtained with syringe, and mixed with a mixture of Anorganic Bovine Bone(ABB) and Autogenous Particulate and allowed to polymerize for 5-10 minutes in order to produce sticky bone.

In the control group

The socket was fully packed with a mixture of Anorganic Bovine Bone(ABB) and Autogenous Particulate (Figure 10).

After fully packing of the socket in both groups gelatin foam was applied then the socket was sutured using a non-resorbable polypropylene suture (figure eight technique for stabilization the gelatin foam) (Figure 13).

Immediately after extraction and socket preservation, a postoperative cone beam-CT (CBCT-1) was ordered for all patient to act as baseline for radiographic analysis. After 6 months all patients were recalled obtaining a second cone beam-CT (CBCT-2) to assess alveolar ridge recession and the amount of bone resorption.

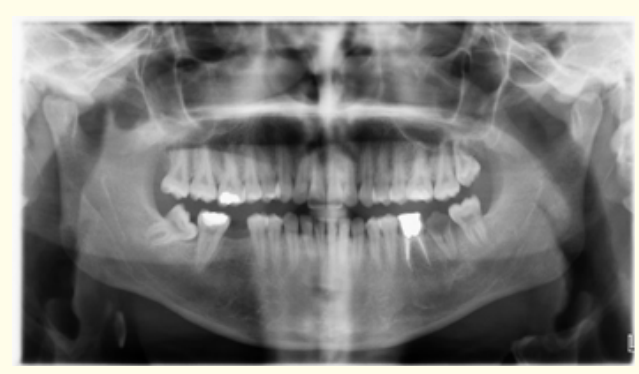

Figure 1: Preoperative panoramic x ray.

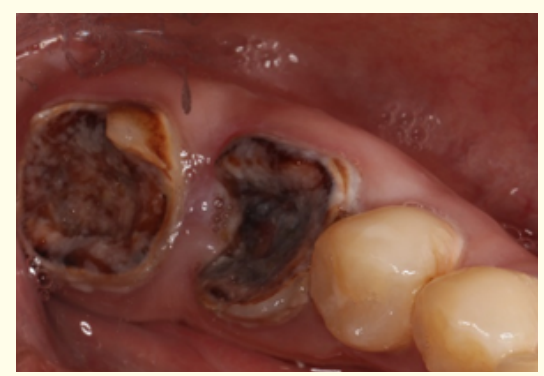

Figure 2: Badly decayed tooth. 


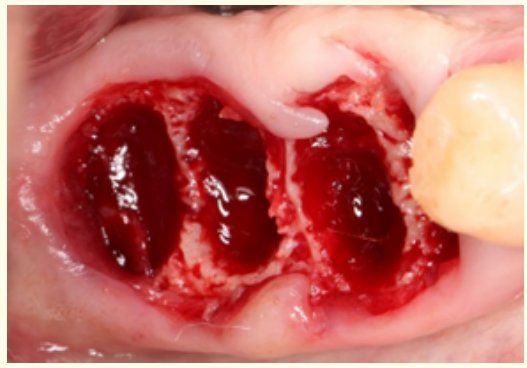

Figure 3: Socket after extraction.

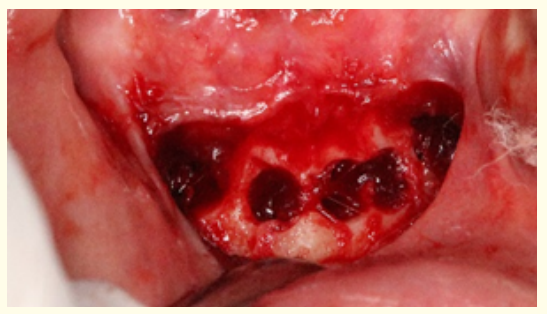

Figure 4: Chin after harvesting autogenous.

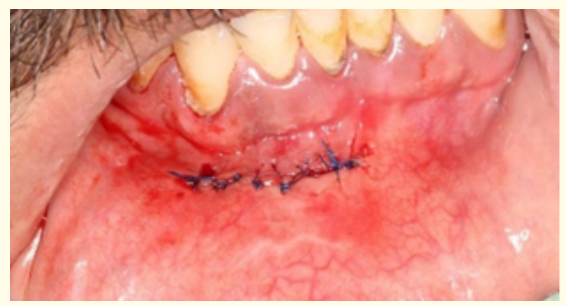

Figure 5: Chin suture.

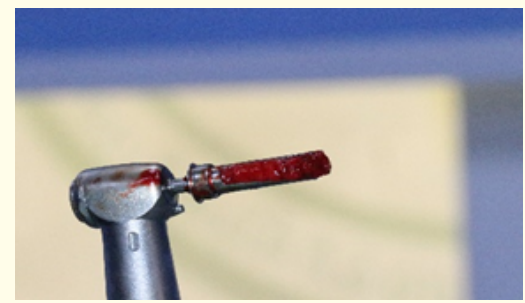

Figure 6: Neo Biotech ACM Auto Chip Maker.

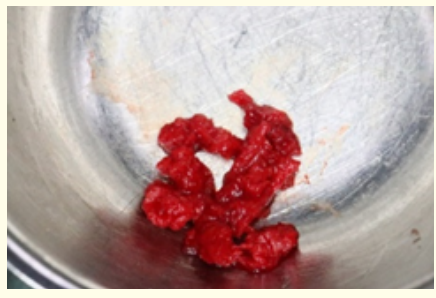

Figure 7: Autogenous bone collected from the chin.
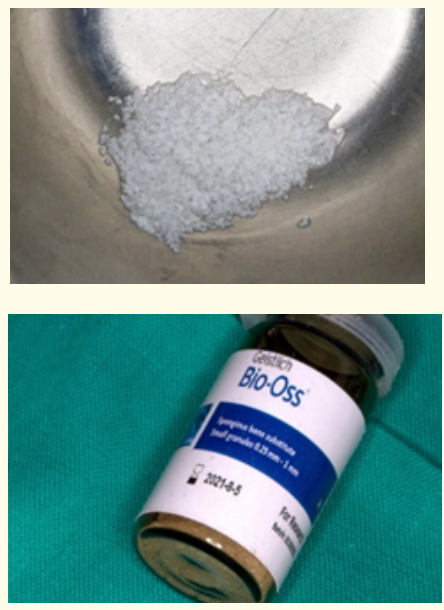

Figure 8: Geistlich Bio-Oss spongious bone substitute small granules $0.25 \mathrm{~mm}-1 \mathrm{~mm}$.

Two types of bone mixtures are used in the present study, namely

- GRoUP A: A Mixture of Anorganic Bovine Bone (ABB) And Autogenous Particulate :which contain as standard a mixture of Geistlich Bio-Oss spongious bone substitute small granules $0.25 \mathrm{~mm}-1 \mathrm{~mm}$ and Autogenous bone particulates harvested from the chin by Neo Biotech ACM Auto Chip Maker $4.5 \mathrm{~mm}$ diameter, $14 \mathrm{~mm}$ length

- GROUP B: A Mixture Of Injectable Platelets Rich Fibrin, ABB And Autogenous Particulates (sticky bone):which contain a mixture between injectable platelets rich fibrin (using 10 cc syringe, 21 gauze needle, PRF tube and a centrifuge at 2500rpm for 2 minutes), and Geistlich Bio-Oss spongious bone substitute small granules $0.25 \mathrm{~mm}-1 \mathrm{~mm}$ and Autogenous bone particulates harvested from the chin by Neo Biotech ACM Auto Chip Maker 4.5mm diameter, 14 mm length. 

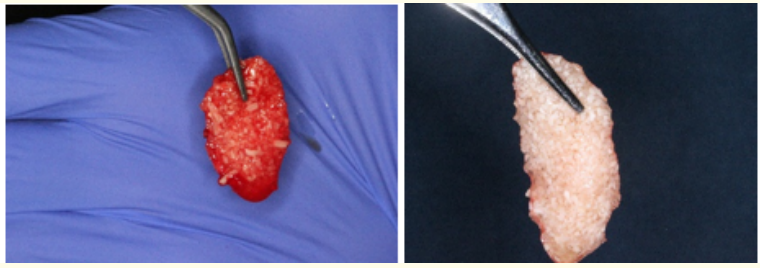

Figure 9: Sticky bone after polymerization.

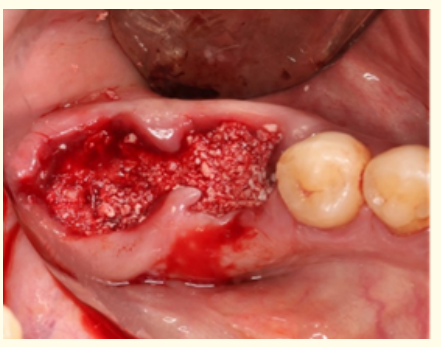

Figure 10: Application of the sticky bone.

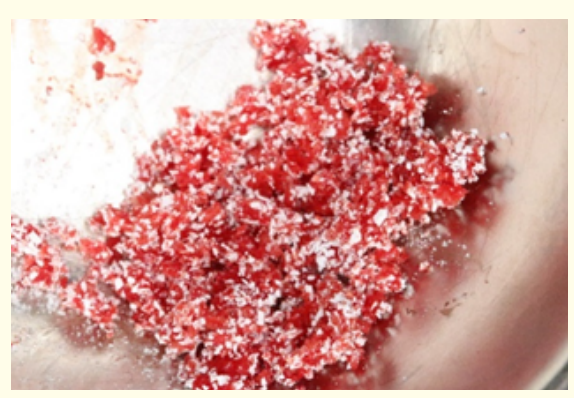

Figure 11: A Mixture of (ABB) And Autogenous Particulate.

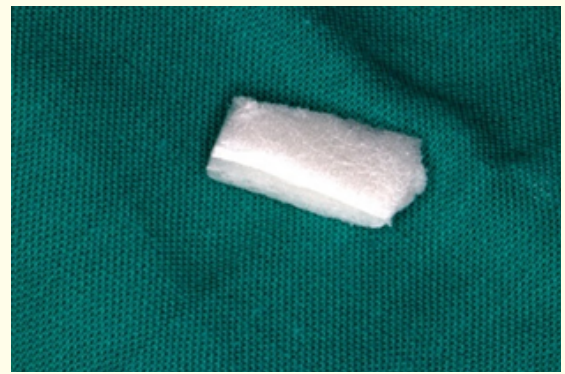

Figure 12: Gelatin foam.

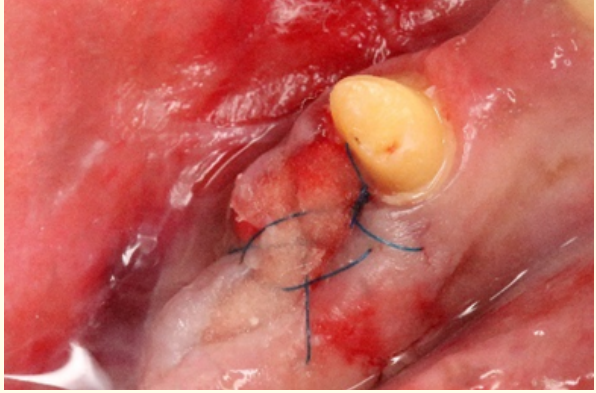

Figure 13: Stabilizing the graft with gelatin foam and suturing the socket.

Post-operative care

- Post-operative medications were prescribed as follows: amoxicillin/clavulanic acid tablets $1 \mathrm{mg}$ every 12 hours for 7 days, diclofenac potassium-sodium 50mg every 8 hours for one week.

- $\quad$ post-operative instruction explained to the patients as follows: Patients were instructed to have liquid or semiliquid diet for the first 3 days after surgery and to gradually return to their normal diet.

- Ice packs for 10 minutes every 30 minutes for 24 hours, strict oral hygiene measures in the form of regular use of the toothbrush and antiseptic mouthwash starting the day after the surgery.

\section{Second stage biopsy harvesting and implant insertion}

Six months after extraction and socket preservation both groups are recalled for biopsy collection and implant placement. for both groups the following steps had taken place. CBCT, clinical intra oral pictures and impression were taken (Figure 14).

One week prior to surgery, a dental hygiene appointment was scheduled, and patients were instructed to use a $0.12 \%$ chlorhexidine oral rinse twice a day for 1 minute. Scrubbing and draping of the patient is carried out in a standard fashion for intra oral procedures. Local anesthesia will be given to the patient infiltration buccal and lingual Mucoperiosteal flap was done for both group's patients (Figure 15). Trephine with inner diameter $3 \mathrm{~mm}$ and outer diameter $4 \mathrm{~mm}$ is used with a depth of 3 to $4 \mathrm{~mm}$ to harvest the 
bone core (Figure 18). Drilling for the implant in a prosthetically driven position and fixture placement (Figure 20). Suture the flap with non-resorbable propylene sutures (interrupted suture) (Figure 21). Panoramic $x$ ray was taken immediately after the implant placement (Figure 22). The biopsy core is removed from the trephine bur. The biopsy core was inserted in a sterile specimen cup contains $10 \%$ buffered formalin covering the core.

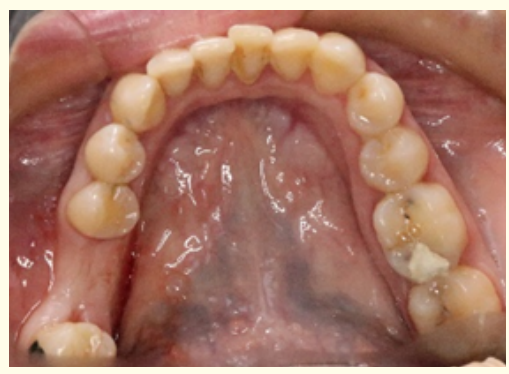

Figure 14: Preoperative occlusal view.

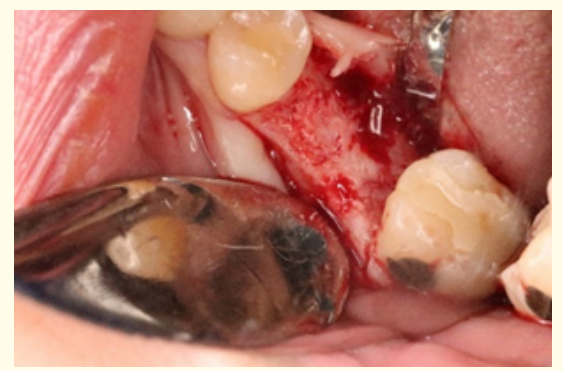

Figure 15: Mucoperiosteal Flap.

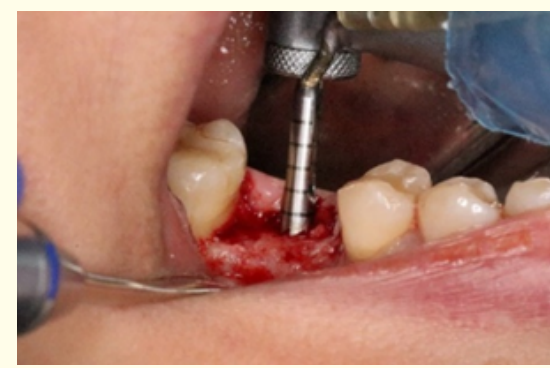

Figure 16: Trephine diameter 3/4mm,Length $10 \mathrm{~mm}$.

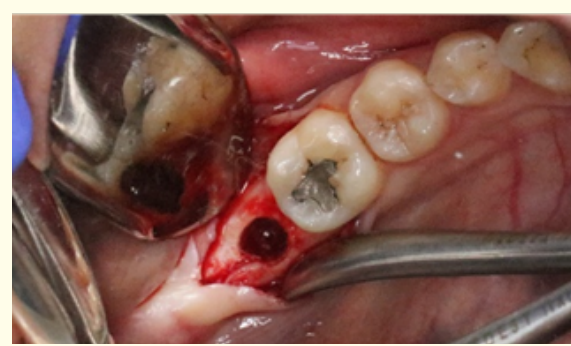

Figure 17: Harvest site.

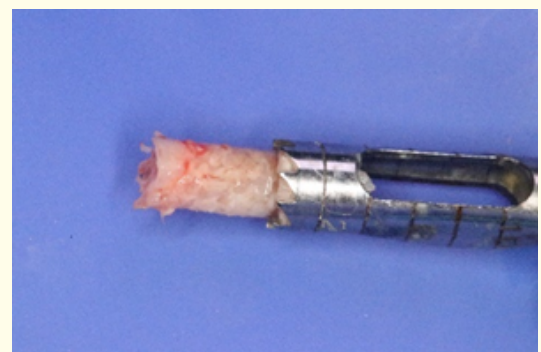

Figure 18: Biopsy core.

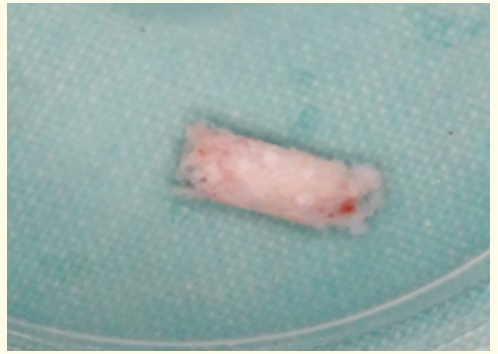

Figure 19: Sterile Specimen cup contains 10\% buffered formalin covering the biopsy.

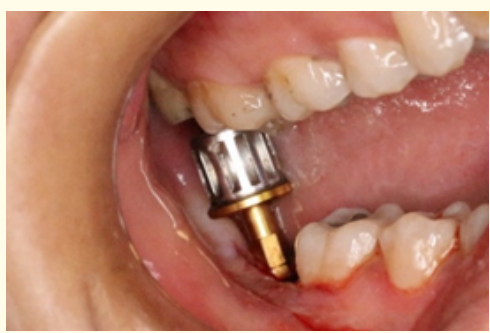

Figure 20: Implant placment. 
Alveolar Ridge Preservation in Mandibular Molars Using Mixture of Anorganic Bovine Bone (ABB) AND Autogenous Particulate vs Mixture of Injectable Platelets Rich Fibrin, ABB and Autogenous Particulates (Sticky Bone) (Randomized Clinical Trial)

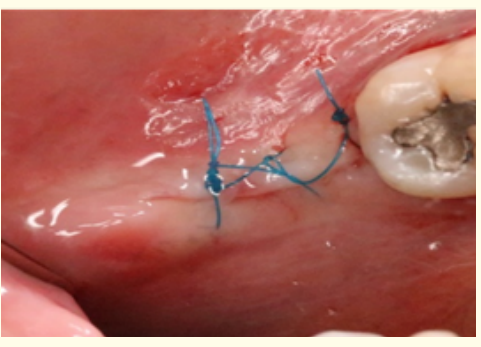

Figure 21: Flap suturing.

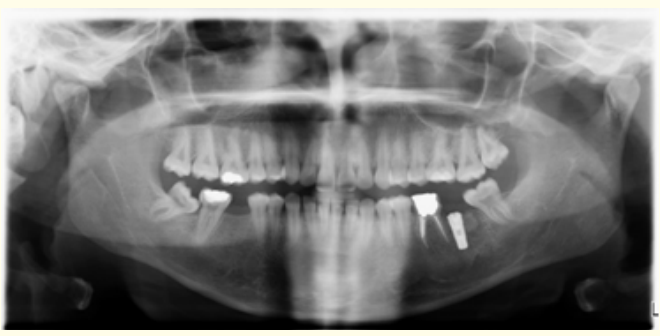

Figure 22: Post operative panoramic x ray.

\section{Postoperative care and follow up}

Postoperatively Patients were instructed to avoid hot foods for 24 hours, and to have liquid or semiliquid diet for the first 3 days after surgery and to gradually return to their normal diet. The sutures were removed one week postoperative. The healing period was monitored to ensure sustained closure of the grafted site and infection-free regeneration.

Patients were prescribed amoxicillin $1 \mathrm{~g}+$ clavulanate potassium $^{1}$, three times per day, for 1 week starting at the day of surgery; metronidazole $500 \mathrm{mg}^{2}$ twice per day for 1 week starting from surgery day; ibuprofen $600 \mathrm{mg}^{3}$, anti-inflammatory/analgesic, twice a day for 2-3 days after surgery; and chlorhexidine $0.12 \%$ mouthwash oral rinses were prescribed twice a day for 2 weeks.

\section{Clinical assessment}

Patients were called for follow-up 1-week post-operative for suture removal and then monthly for six months and were assessed for healing.

\section{Radiographic assessment}

Radiographic assessment of alveolar ridge bone loss

According to prior evidence a close estimate of the bone loss could be measured through a CBCT that is comparable to surgical exploration and can be used for diagnosis of bone defects in periodontal diseases in clinical settings [24].

From both the immediate (CBCT-1) and 6 months post-operative (CBCT-2) using DICOM viewer software ${ }^{5}$, the measurements were calculated and assessed.

The measurements were recorded from the same distance relative to the mental foramen in CBCT- 1 and CBCT- 2 in each case and the differences were calculated to result on the remodeling changes that happened in the alveolar ridge (Figure 23, Figure 24).

\section{Results}

Clinical results

- A total of 20 patients (8males, 12 females; age range of 18 to 60 years old) were enrolled in this study, the patients were randomly divided into 2 equal groups using block randomization with stratification (block size: 4) using a formula on Microsoft Excel Software. the mean follow-up was of 9 months from the extraction and socket preservation (first stage) (Figure 26).

- 20 completely successful cases were recorded, no failures, accidents, adverse event nor poor results were recorded. All extractions healed well and the patients were visiting the clinic every week for four weeks.

\footnotetext{
${ }^{1}$ Augmenting 1gm, Medical Union Pharmaceuticals (MUP) - Cairo, Egypt, GlaxoSmithKline(gsk) S.A.E. - A.R.E.

${ }^{2}$ Flagyl Sanofi Aventis, Cairo, Egypt.

${ }^{3}$ Brufen, Boots, Cairo, Egypt.

${ }^{4}$ Hexitol, The Arab Drug Company, Cairo, Egypt.

${ }^{5}$ Blue Sky Bio, Blue Sky Bio, LLC, U.S.A
}

Citation: Mohamed Darwish Elsayed Darwish., et al. "Alveolar Ridge Preservation in Mandibular Molars Using Mixture of Anorganic Bovine Bone (ABB) AND Autogenous Particulate vs Mixture of Injectable Platelets Rich Fibrin, ABB and Autogenous Particulates (Sticky Bone) (Randomized Clinical Trial)". Acta Scientific Orthopaedics 4.2 (2021): 31-50. 


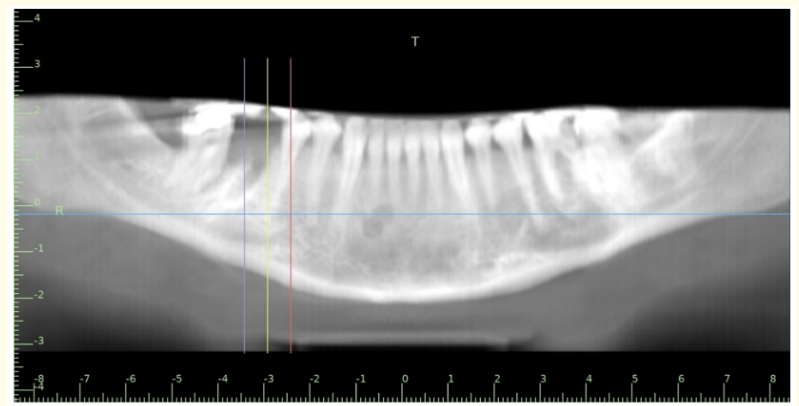

(a)

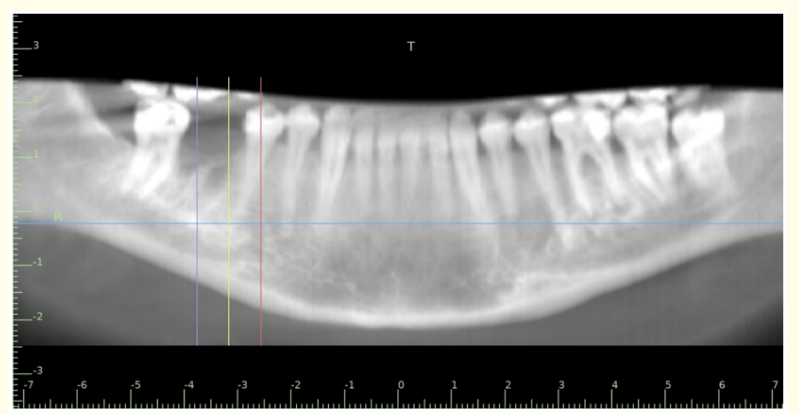

(b)

Figure 23: A referenced point of the line coincides with the mental foramen) chosen for identical measurement point between CBCT-1 (a) and CBCT-2(b) ( blue line cross sectional view showing mental foramen while the measured alveolar socket coincides with the yellow line and the distance between the two cross sections is recorded in CBCT-1 and duplicated in CBCT-2 for calculating differences in ridge height and width at the same point).

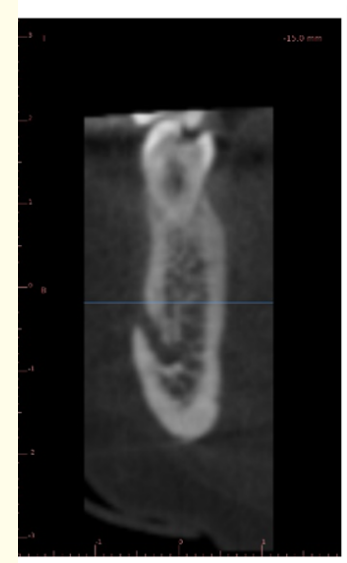

(a)

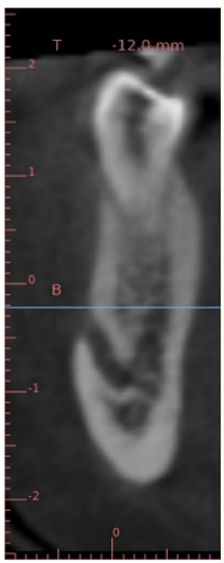

(b)
Figure 24: The cross-sectional views, (CBCT-1) (a) and (CBCT-2)

(b) with recorded distance in relevance to the mental foramen.

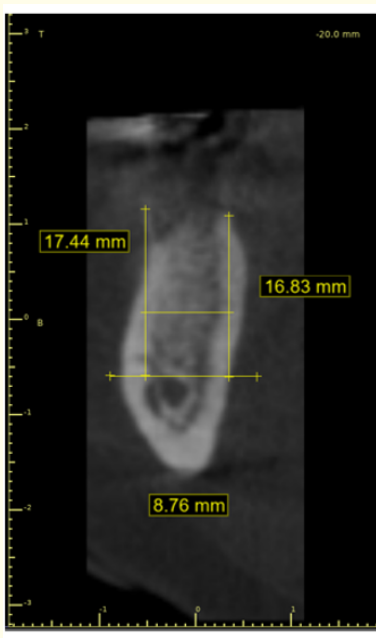

(a)

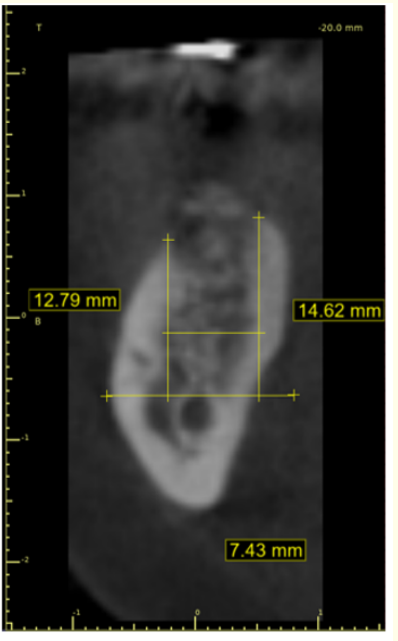

(b)
Figure 25: Measurements applied on CBCT-1 (a) and CBCT-2 (b) at the same point of the ridge for remodeling calculation.

- $\quad$ CBCT radiography was performed six months after tooth extraction suggested good healing of the sockets (Figure 37).

- Implants were rehabilitated with screw retained crowns and no detachments of the prostheses were reported during the evaluation period.

- At every follow-up visit, the rehabilitations were checked for signs of peri-implant inflammation, radiographic signs of bone loss, fractures, and mobility of the prosthesis or of abutments, none of which was recorder for all the 20 patients.

- Soft tissue contours showed no significant changes neither after the first stage surgery nor after the second stage surgery.

- Examination showed maintained bone levels around implant platforms was unchanged after 3 month of implant placement.

Radiographic results

Variation of height of buccal alveolar crest

Comparison between Autogenous + ABB graft group and sticky bone group: 


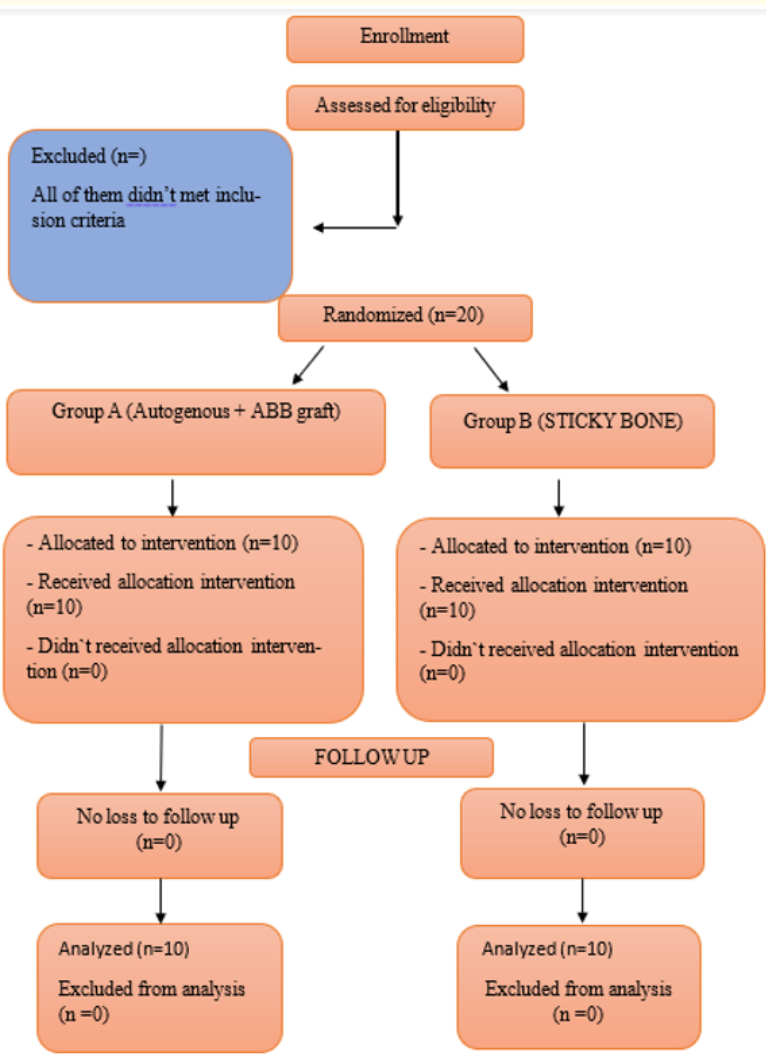

Figure 26: Flow diagram of the study.

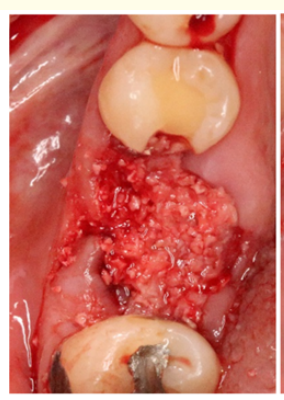

(a)

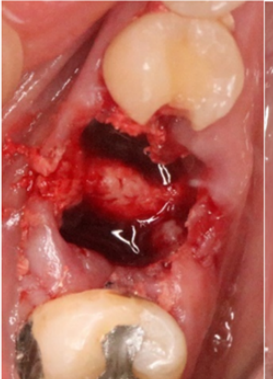

(b)

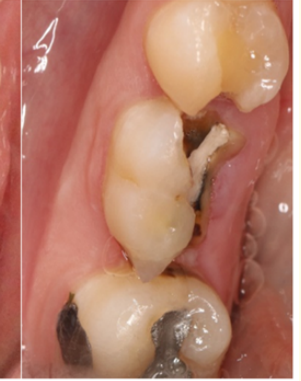

Figure 27: (a) Badly decayed tooth. (b) Socket after extraction. (c) Application of the mixture inside the socket.

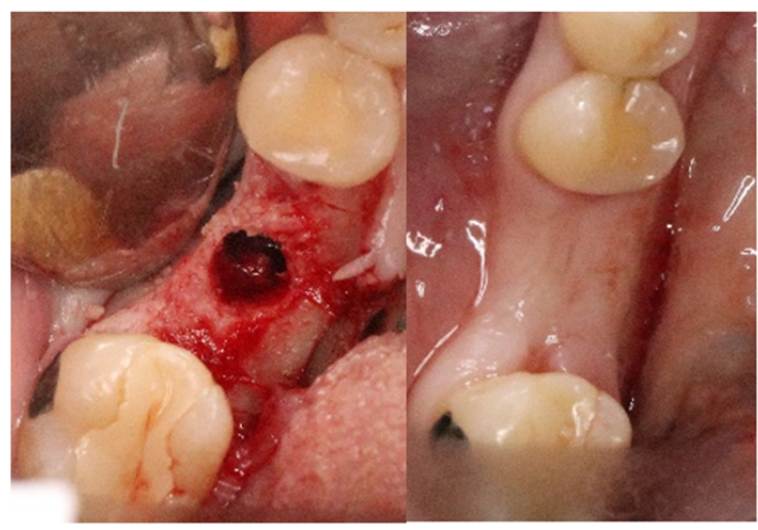

(a)

(b)

Figure 28: (a) Occlusal view showing good healing after 6 months. (b) Occlusal view showing the ridge with a hole made by the trephine bur.

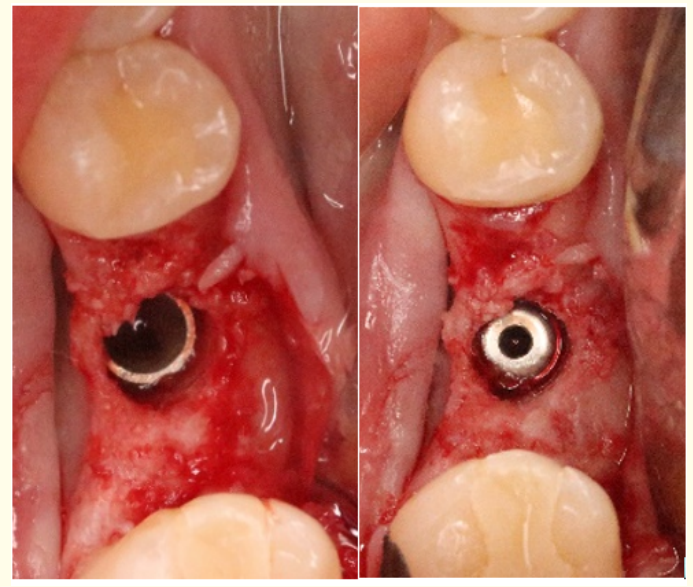

Figure 29: Showing the placement of the dental implant.

(a)

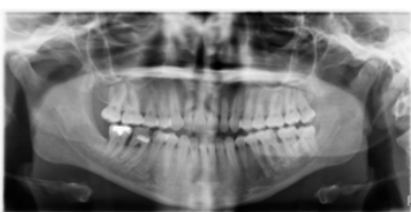

Figure 30: (a) Panoramic $x$ ray showing badly decayed tooth no 46. (b) Panoramic x ray immediately taken after implant placement tooth no 46. 
Alveolar Ridge Preservation in Mandibular Molars Using Mixture of Anorganic Bovine Bone (ABB) AND Autogenous Particulate vs Mixture of Injectable Platelets Rich Fibrin, ABB and Autogenous Particulates (Sticky Bone) (Randomized Clinical Trial)

The lowest variation value of the height of buccal alveolar crest was recorded in the sticky bone group and the highest variation value was recorded in (Autogenous + ABB graft) group. Unpaired t-test revealed that the difference was not statistically significant $(\mathrm{P}=0.6018)$ (Table 1$)$.

\begin{tabular}{|c|c|c|}
\hline P.o.C & $\begin{array}{c}\text { Autogenous + ABB } \\
\text { graft }\end{array}$ & Sticky bone \\
\hline Mean & 2.64 & 2.34 \\
\hline Std Dev & 1.48 & 1.00 \\
\hline Std error & 0.49 & 0.33 \\
\hline Max & 5.36 & 4.53 \\
\hline Min & 0.95 & 0.83 \\
\hline t-value & \multicolumn{2}{|c|}{0.5311} \\
\hline P-value & $0.6018 *$ \\
\hline
\end{tabular}

Table 1: Variation of height of buccal alveolar crest in Autogenous+ ABB graft group vs Sticky bone group, and significance of the difference using unpaired t-test.

* (NS) Not significant, $\mathrm{P}$ value $>0.05$.

Variation of height of lingual alveolar crest

Comparison between Autogenous + ABB graft group and sticky bone group:

The lowest variation value of the height of lingual/palatal alveolar crest was recorded in the sticky bone group and the highest variation value was recorded in (Autogenous + ABB graft) group. Unpaired t-test revealed that the difference was not statistically significant $(\mathrm{P}=0.3682)$ (Table 2$)$.

\begin{tabular}{|c|c|c|}
\hline P.o.C & Autogenous + ABB graft & Sticky bone \\
\hline Mean & 1.59 & 1.27 \\
\hline Std Dev & 0.86 & 0.68 \\
\hline Std error & 0.29 & 0.23 \\
\hline Max & 3.57 & 2.67 \\
\hline Min & 0.57 & 0.23 \\
\hline t-value & \multicolumn{2}{|c|}{0.9230} \\
\hline P-value & \multicolumn{2}{|c|}{$0.3682^{*}$} \\
\hline
\end{tabular}

Table 2: Variation of height of lingual alveolar crest in

Autogenous+ ABB graft group vs Sticky bone group, and significance of the difference using unpaired t-test.

* (NS) Not significant, $\mathrm{P}$ value $>0.05$.
Variation of width of alveolar crest

Comparison between Autogenous + ABB graft group and sticky bone group:

The lowest variation value of the width of alveolar crest was recorded in the sticky bone group and the highest variation value was recorded in (Autogenous + ABB graft) group. Unpaired t-test revealed that the difference was not statistically significant $(\mathrm{P}=$ 0.7173) (Table 3).

\begin{tabular}{|c|c|c|}
\hline P.o.C & Autogenous + ABB graft & Sticky bone \\
\hline Mean & 1.63 & 1.48 \\
\hline Std Dev & 0.97 & 0.85 \\
\hline Std error & 0.32 & 0.28 \\
\hline Max & 3.59 & 2.73 \\
\hline Min & 0.22 & 0.05 \\
\hline t-value & \multicolumn{2}{|c|}{0.3678} \\
\hline P-value & \multicolumn{2}{|c|}{$0.7173^{*}$} \\
\hline
\end{tabular}

Table 3: Variation of width of alveolar crest in Autogenous+ ABB graft group vs Sticky bone group, and significance of the difference using unpaired t-test.

* (NS) Not significant, P value $>0.05$.

In conclusion

The variation value of the buccal alveolar crest was lower in the sticky bone group $(2.34 \pm 1.00)$ compared with the Autogenous $+A B B$ group $(2.64 \pm 1.48)$ (Table 1$)$. The variation value of the height of the lingual/palatal alveolar crest was lower in the sticky bone $(1.27$ \pm 0.68 ) compared with the Autogenous + ABB group group $(1.59 \pm$ 0.86 ) (Table 2). The variation value of the width of the alveolar crest was lower in the sticky bone group $(1.48 \pm 0.85)$ compared with the Autogenous+ABB group $(1.63 \pm 0.97)$ (Table 3 , Figure 31).

\section{Histological and Histomorphometric analysis}

Bone area percent

Comparison between Autogenous + ABB graft group and sticky bone group: 


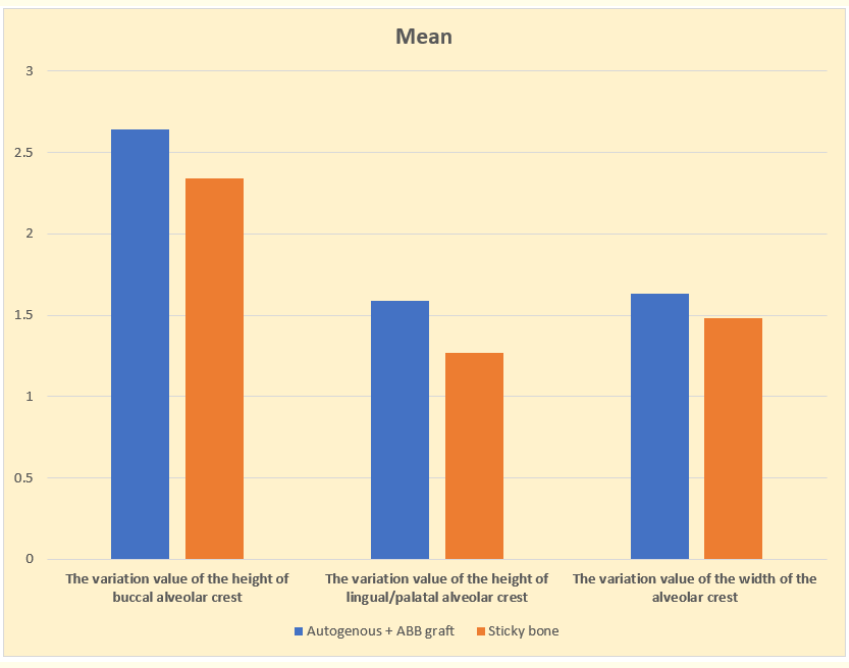

Figure 31: Column chart showing mean variation values of the buccal and lingual/palatal alveolar crest height and the alveolar crest width in the two groups.

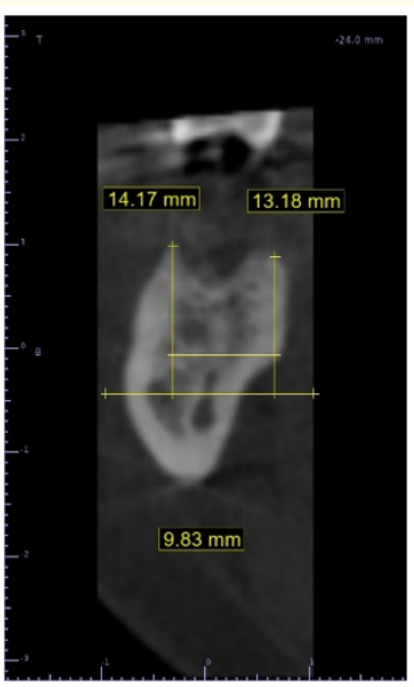

(a)

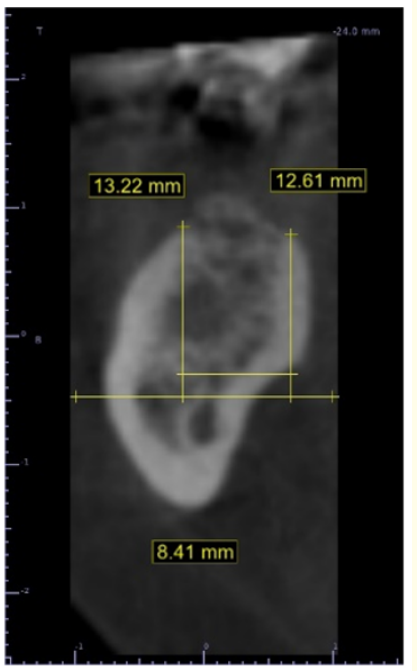

(b)
Figure 32: (Group A), CBCT cross section view showing the differences between the height (buccal and lingual) and the width of the socket, (a) CBCT-1 Immediately after extraction and socket preservation (b) CBCT-2 after 6 months.

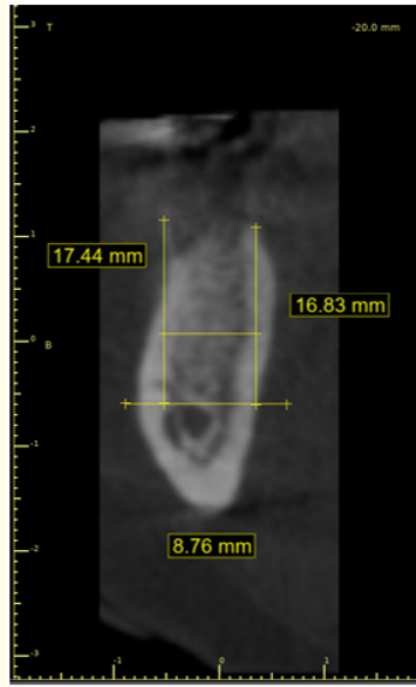

(a)

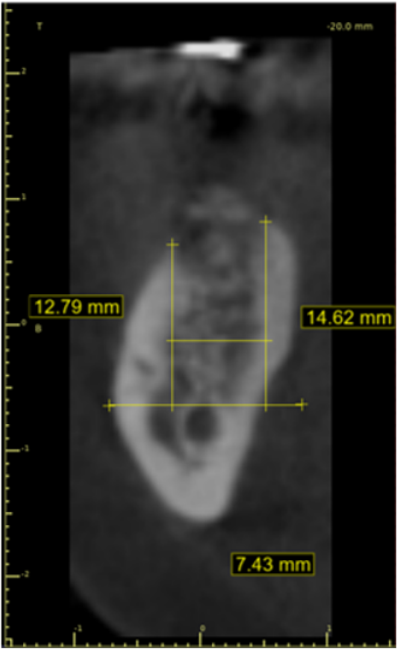

(b)
Figure 33: (Group B), CBCT cross section view showing the differences between the height (buccal and lingual) and the width of the socket, (a)CBCT-1 Immediately after extraction and socket preservation (b) CBCT-2 after 6 months.

Sticky bone group revealed a higher mean area percent of bone compared to the mean value of (Autogenous + ABB graft) group. Unpaired t-test revealed that the difference was statistically significant $(\mathrm{P}=0.0012)$ (Table 4, Figure 34).

\begin{tabular}{|c|c|c|}
\hline P.O.C & Autogenous + ABB graft & Sticky bone \\
\hline Mean & 42.34 & 57.92 \\
\hline Std Dev & 8.67 & 9.43 \\
\hline Std error & 2.89 & 3.15 \\
\hline Max & 57.44 & 69.78 \\
\hline Min & 30.58 & 42.44 \\
\hline t-value & 3.8461 \\
\hline P-value & 0.0012 \\
\hline
\end{tabular}

Table 4: Bone area percent of Autogenous + ABB graft group vs Sticky bone group, and significance of the difference using unpaired t-test.

*significant at $\mathrm{p}<0.05$. 


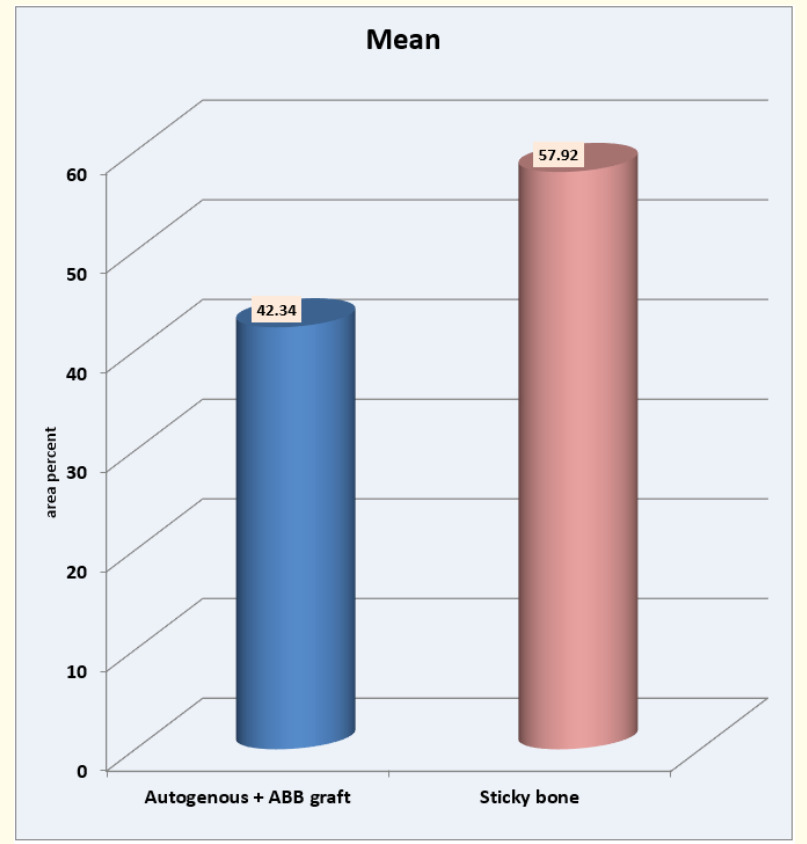

Figure 34: Column chart showing mean area percent of bone between (Autogenous + ABB graft) group and sticky bone group.

\section{Area percent of residual material}

Comparison between Autogenous + ABB graft group and sticky bone group:

(Autogenous + ABB graft) group revealed a higher mean area percent of residual material compared to the mean value of sticky bone group. Unpaired t-test revealed that the difference was not statistically significant $(P=0.1903)$ (Table 5, Figure 35).

\begin{tabular}{|c|c|c|}
\hline P.o.C & Autogenous + ABB graft & Sticky bone \\
\hline Mean & 32.19 & 29.11 \\
\hline Std Dev & 3.22 & 6.39 \\
\hline Std error & 1.07 & 2.13 \\
\hline Max & 37.87 & 36.23 \\
\hline Min & 27.22 & 17.54 \\
\hline t-value & \multicolumn{2}{|c|}{1.3612} \\
\hline P-value & $0.1903^{*}$ \\
\hline
\end{tabular}

Table 5: Area percent of residual material in Autogenous+ ABB graft group vs Sticky bone group, and significance of the difference using unpaired t-test.

* (NS) Not significant, P value $>0.05$.

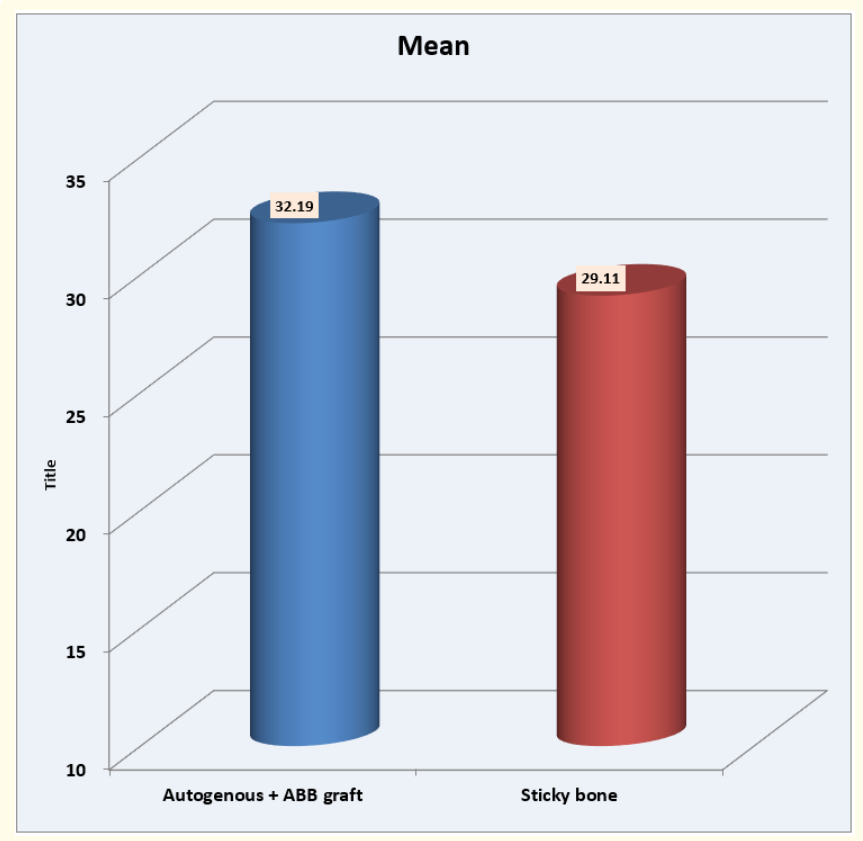

Figure 35: Column chart showing mean area percent of residual material between (Autogenous + ABB graft) group and sticky bone group.

Histomporhometric microscopic pictures

Autogenous + ABB graft group:

- $\quad H \& E$ Histological Microscopic picture (Magnification X100) demonstrating extensive xenograft remnants surrounded cellular fibrous stroma and an adjacent area of lamellar bone trabeculae (Figure 36).

- H\&E Histological Microscopic picture (Magnification X200) demonstrating xenograft remnants surrounded cellular fibrous stroma and an adjacent area of lamellar bone trabeculae (Figure 37).

\section{Sticky Bone group}

- H\&E Histological Microscopic picture of Magnification X100 demonstrating xenograft remnants surrounded by irregular newly formed trabeculae of woven bone and cellular fibrous stroma (Figure 38). 


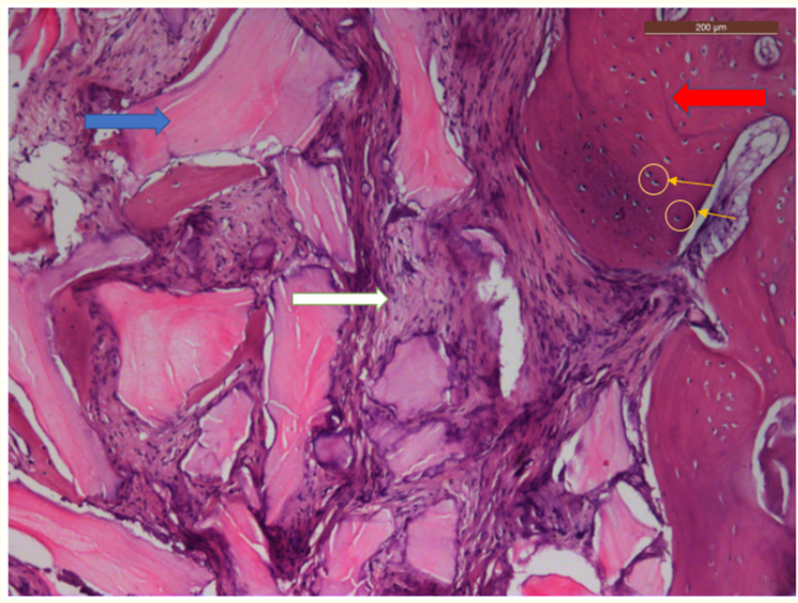

Figure 36: H\&E Histological Microscopic picture of Autogenous + ABB graft group (Magnification X100) demonstrating.

A) Extensive xenograft remnants (blue arrow).

B) Cellular fibrous stroma (white arrow).

C) Adjacent area of lamellar bone trabeculae (red arrow).

D)Osteocyte cells (yellow circles).

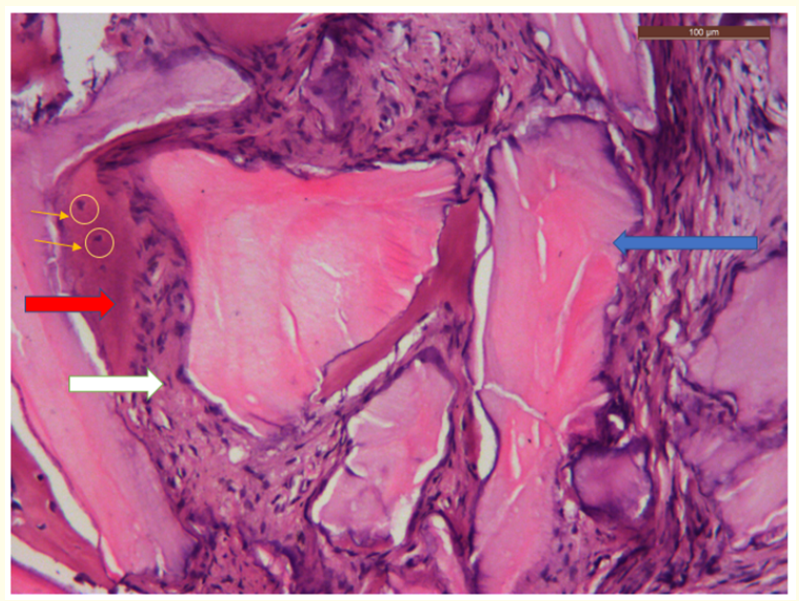

Figure 37: H\&E Histological Microscopic picture of Autogenous + ABB graft GROUP.(Magnification X200) demonstrating.

A) Xenograft remnants (blue arrow).

B) Cellular fibrous stroma (white arrow).

C)Trabeculae of lamellar bone (red arrow).

D)Osteocytes cells (yellow circles).

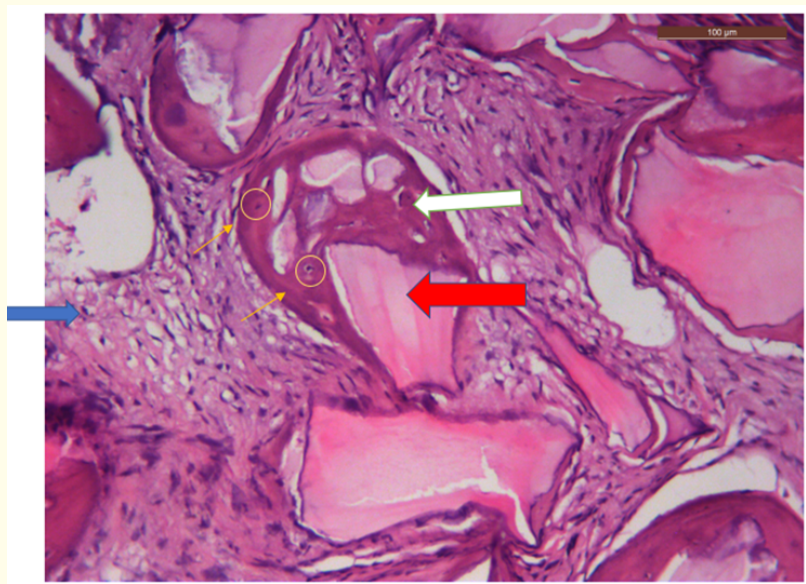

Figure 38: H\&E Histological Microscopic picture of Sticky bone group. (Magnification X200) demonstrating.

A) Xenograft remnants (red arrow).

B) Irregular newly formed trabeculae of woven bone

(white arrow).

C) Cellular fibrous stroma (blue arrow).

D)Osteocyte cells (yellow circles).

- H\&E Histological Microscopic picture of Magnification X200 demonstrating well-formed extensive trabeculae of lamellar bone showing multiple reversal lines and areas of marrow spaces (Figure 39).

\section{Discussion}

Dental implants have become the standard of care for cases of tooth extraction, sometimes for some reasons (financial or medical) the patients postponed the treatment, the need of good functionality and aesthetics is depending on the residual soft and hard tissue after tooth extraction, both of them are important in order to achieve long lasting and successful dental implant.

Socket preservation is done after tooth extraction by using biomaterials:, Autogenous, allogeneic, xenogeneic, and alloplastic, iPRF. or a mixture between two or more materials to take the advantage of each one [25].

The aim of this study was a Radiographic and Histologic assessment using a Mixture of Anorganic Bovine Bone (ABB) and Autog-

Citation: Mohamed Darwish Elsayed Darwish., et al. "Alveolar Ridge Preservation in Mandibular Molars Using Mixture of Anorganic Bovine Bone (ABB) AND Autogenous Particulate vs Mixture of Injectable Platelets Rich Fibrin, ABB and Autogenous Particulates (Sticky Bone) (Randomized Clinical Trial)". Acta Scientific Orthopaedics 4.2 (2021): 31-50. 


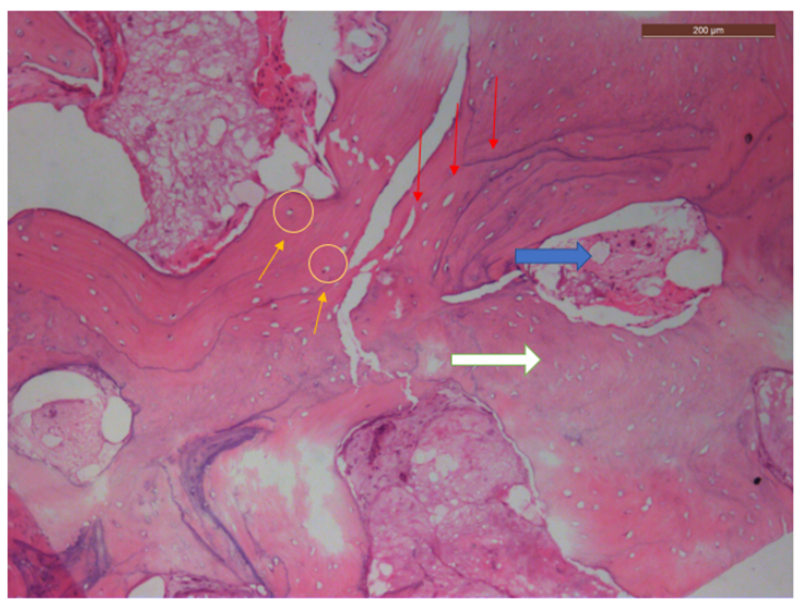

Figure 39: H\&E Histological Microscopic picture of Sticky bone group. (Magnification X100) demonstrating.

A) Well-formed extensive trabeculae of lamellar bone (white arrow)

B) Multiple reversal lines (red arrows)

C) Areas of marrow spaces (blue arrow)

D) Osteocyte cells (yellow circles).

enous Particulate vs a Mixture of Injectable Platelets Rich Fibers, $\mathrm{ABB}$ and Autogenous Particulates (sticky bone) In socket preservation.

A total of 20 patients (8males, 12 females; age range of 18 to 60 years) was enrolled in this study, The patients were randomly divided into 2 equal groups using block randomization with stratification (block size: 4) using a formula on Microsoft Excel Software. the mean follow-up period was 9 months from the extraction and socket preservation (first stage).

20 completely successful cases were recorded, no failures, accidents, adverse event nor poor results were recorded. All extractions healed well and the patients were visiting the clinic every week for four weeks.

The flapless tooth extraction is generally performed to obtain a reduction of healing time and discomfort, in this study all the extractions and socket preservation procedure were flapless to prevent disruption of the blood supply to the bone which results in decreased the healing time and predictable results compared to the flap approach.

(Barone., et al. 2014 [26]), in a recent RCT showed that the flapless approach for ridge preservation was more successful than the flap approach in preserving horizontal ridge dimension and width of keratinized tissues.

A recent systematic review on ridge preservation with a flapless approach showed that bone dimensional changes were lower for flapless approach compared with a flap approach [27].

Numerous studies have shown the extent of vertical and/or horizontal site collapse after tooth extraction when no socket preservation/augmentation procedures have been performed. (Iasella JM., et al. 2003 [28]) compared bucco-lingual width collapse after extraction; when no bone replacement was used the average socket decreased in bucco-lingual width from $9.1 \mathrm{~mm}$ to $6.4 \mathrm{~mm}$. in our study The variation value of the width of the alveolar crest was lower in the sticky bone group $(1.48 \pm 0.85)$ compared with the Autogenous $+\mathrm{ABB}(1.63 \pm 0.97)$ the difference was not statistically significant $(\mathrm{P}=0.7173)$ (Figure).

(Van Der Weijden F., et al. 2009 [29]) in a systematic review with evaluation periods varying from 3 to 12 months. During the post extraction healing period, the mean changes as based on the data derived from the selected studies show the clinical loss in width radiographically $(3.87 \mathrm{~mm})$ and the loss in height, assessed both clinically (1.67-2.03 $\mathrm{mm}$ ) as well as radiographically $(1.53 \mathrm{~mm})$.

In this study the evaluation period was 6 months. The variation value of the buccal alveolar crest was lower in the sticky bone group $(2.34 \pm 1.00)$ compared with the Autogenous+ABB $(2.64 \pm$ 1.48) (Figure 35). The variation value of the height of the lingual/ palatal alveolar crest was lower in the sticky bone $(1.27 \pm 0.68)$ compared with the Autogenous+ABB group (1.59 \pm 0.86$)$.

(Tatullo M., et al. 2012 [30]), claimed that there is an osteoconductive capacity of PRF used as osteoregenerative material.

In our study the sticky bone group showed histologically wellformed trabeculae of lamellar bone and areas of marrow spaces (Magnification X200). 
In our study the use of a mixture of Injectable Platelets Rich Fibrin, ABB and Autogenous Particulates (sticky bone) gives a moldable, well adapted graft over various shapes of bony defect [31].

In this study Sticky bone group revealed a higher mean area percent of bone compared to the mean value of (Autogenous + ABB graft) group. Unpaired t-test revealed that the difference was statistically significant $(P=0.0012)$, Autogenous $+A B B$ graft group showed mean area percent of bone equels $42.34 \%$, the sticky bone group showed mean area percent of bone equels $57.92 \%$ with a significant value $\mathrm{p}<0.05$.

On the other hand (Scarano A., et al. 2006 [32]) claimed that, Bio-Oss $\AA$ [Geistlich Pharma AG, Wohlhusen, Switzerland] and hydroxyapatite were surrounded by bone, compared with autogenous bone, they show smaller areas of regenerated bone

In this study (Autogenous + ABB graft) group revealed a higher mean area percent of residual material compared to the mean value of sticky bone group. Unpaired t-test revealed that the difference was not statistically significant $(P=0.1903)$.

(Autogenous + ABB graft) group showed higher mean area percent of residual material equals $32.19 \%$ compared to the mean value of the sticky bone group $29.11 \%$ which was Not significant, with $\mathrm{P}$ value $>0.05$, thanks to the common biomaterial between the two groups (ABB), Conflicting data are present in the literature about the performance of anorganic bovine bone (ABB). (Zaffe D., et al. 2005 [33]) in the long term Histolomorphometric analysis, studies have shown that ABB has faster resorption in the initial healing period after graft insertion, (Traini T., et al. 2007 [34]) said that the resorption rate is slowed down in subsequent time periods.

(Guarnieri R., et al. 2018 [35]) in a case report, he used Histomorphometric analysis of bone regeneration with bovine grafting material after 24 months of healing, results was Newly formed bone presented of different levels of maturation and numerous osteocytes, with greater numbers in bone closer to the grafted particles $(27.3 \%$ vs. $11.2 \%, \mathrm{p}<0.05)$. The Histomorphometry analysis showed $40.84 \%$ of newly formed bone, $33.58 \%$ residual graft material, however ABB underwent considerable resorption, a big amount of grafting material was still exist after two years of socket healing. This study clarified that the bovine bone grafts are classified as long-term degradation bone graft material.

Bio-Oss is an anorganic bovine bone substitute (ABB) with osteoconductive properties and high biocompatibility (Jen- Sen., et al. 1996). It has been tested in several randomized clinical trials registered in the Cochrane Library and is that $\mathrm{ABB}$ is one of the best biomaterial. Controversy remains, however, (Piattelli M., et al. 2019 [36]), asked is this graft source is truly resorbable?

(Fujioka-Kobayashi M., et al. 2017 [37]) the added number of cells contained within fibrin matrix (liquid form of PRF) was further shown to release higher total growth factor release when compared to control L-PRF.

(Miron RJ., et al. 2017 [37]) claimed that i-PRF showed the potential to contain a number of growth factors responsible for tissue regeneration capable of inducing fibroblast behavior.

(Daugela P., et al. 2018 [39]) found that clinical and Experimental studies have found that PGF, such as the TGF $\beta-1$ and FGF, enhance bone formation during bone healing.

\section{Summary}

Socket preservation following tooth extraction has shown high success rates regarding to the preservation of in the preservation ofthe ridge height and width. Mand minimizinge ridge collapse due to normal remodeling and ridge collapse.

Several grafting materials have been proposed in the literature in order to augment the socket after tooth extraction.

This present study was conducted to radiographically and histologically assess the usage of a mixture of Anorganic Bovine Bone (ABB) And Autogenous Particulate vs a mixture of Injectable Platelets Rich Fibers, $\mathrm{ABB}$ and Autogenous Particulates (sticky bone) in socket preservation.

This was a randomized clinical trial conducted on 20 patients to evaluate if the socket preservation of the alveolar ridge in the lower molars using a mixture of Anorganic Bovine Bone(ABB) and Autogenous Particulate gives better results regarding the quality of bone and the dimension of preservation of the socket than a mixture of Injectable Platelets Rich Fibrin, ABB And Autogenous particulate bones (sticky bone). 
Alveolar Ridge Preservation in Mandibular Molars Using Mixture of Anorganic Bovine Bone (ABB) AND Autogenous Particulate vs Mixture of Injectable Platelets Rich Fibrin, ABB and Autogenous Particulates (Sticky Bone) (Randomized Clinical Trial)

AAll patients were recalled 2 days after the operation then weekly for one month then monthly until the implant placement 6 months postoperative. The clinical evaluation included assessment of postoperative sequelae including edema, pain and postoperative healing. Radiographic evaluation involved CBCT radiographs taken for every patient immediately postoperative and 6 months postoperatively to evaluate crestal bone height (buccally and lingually) and width variations.

The radiographic results showed that the variation value of the buccal alveolar crest was lower in the sticky bone group $(2.34$ $\pm 1.00)$ compared with the Autogenous+ABB $(2.64 \pm 1.48)$. (The variation value of the height of the lingual/palatal alveolar crest was lower in the sticky bone $(1.27 \pm 0.68)$ compared with the Autogenous+ABB group $(1.59 \pm 0.86)$. The variation value of the width of the alveolar crest was lower in the sticky bone group $(1.48 \pm 0.85)$ compared with the Autogenous+ABB group (1.63 \pm 0.97 ) for all the radiographic results statically not significant, $P$ value $>0.05$ (Figure 26).

The histomorphometric results showed: the sticky bone group revealed a higher mean area percent of bone compared to the mean value of Autogenous + ABB graft group. Unpaired t-test revealed that the difference was statistically significant $(P=0.0012)$. Autogenous + ABB graft group showed a higher mean area percent of residual material compared to the mean value of sticky bone group. Unpaired t-test revealed that the difference was not statistically significant $(\mathrm{P}=0.1903)$.

This study showed statistical significance between the two groups, the sticky bone group and the Autogenous + ABB graft group in the mean area percent of bone, and showed no statistical significance in all other results.

This study showed in the microscopic pictures

(Autogenous + ABB graft) group

- Magnification X100: Demonstrated extensive xenograft remnants surrounded cellular fibrous stroma and an adjacent area of lamellar bone trabeculae

- $\quad$ Magnification X200: Demonstrated xenograft remnants surrounded cellular fibrous stroma.
Sticky bone group

- Magnification X100: Demonstrated well-formed trabeculae of lamellar bone and areas of marrow spaces.

- Magnification X200: Demonstrated a well-formed trabecula of lamellar bone and areas of marrow spaces.

\section{Conclusion}

Socket preservation after tooth extraction is a very successful and simple technique with respect to some guidelines followed by the operator and the patient. The results are predictable in preserving the socket from collapse which will result in a successful prosthetically driven dental implant in the second stage surgery.

Mixture of Injectable Platelets Rich Fibrin, ABB and Autogenous Particulates (sticky bone). showed a statistical significance regarding the mean value of bone area percent compared to a mixture of ABB and Autogenous bone. Sticky bone is better than the mixture of $\mathrm{ABB}$ and Autogenous bone in amount of bone formation.

\section{Bibliography}

1. Dds DS., et al. "Alveolar ridge preservation using an open membrane approach for sockets with bone deficiency: A randomized controlled clinical trial". Clinical Implant Dentistry (2019): 175-182.

2. Report C. "Clinical and Radiographic Evaluation of Socket Preservation Using Autologous Concentrated Growth Factors Enriched Bone Graft Matrix (Sticky Bone): A Case Report". 4 (2016): 1128-1135.

3. Tadjoedin ES., et al. "Deproteinized cancellous bovine bone (Bio-OssR) as bone substitute for sinus floor elevation. A retrospective, histomorphometrical study of five cases". Journal of Clinical Periodontology 30.3 (2003): 261-270.

4. Seibert JS and Salama H. "Alveolar ridge preservation and reconstruction". Periodontology 11.1 (2000): 69-84.

5. Giannoudis P V., et al. "Bone substitutes: an update". Injury 36 (2005).

6. Laurencin C., et al. "Bone graft substitutes". Expert Review of Medical Devices 3.1 (2006): 49-57. 
Alveolar Ridge Preservation in Mandibular Molars Using Mixture of Anorganic Bovine Bone (ABB) AND Autogenous Particulate vs Mixture of Injectable Platelets Rich Fibrin, ABB and Autogenous Particulates (Sticky Bone) (Randomized Clinical Trial)

7. Kumar P., et al. "Bone grafts in dentistry". Journal of Pharmacy and Bioallied Sciences 5 (2013).

8. Artzi Z and Nemcovsky CE. "The Application of Deproteinized Bovine Bone Mineral for Ridge Preservation Prior to Implantation. Clinical and Histological Observations in a Case Report”. Journal of Periodontology 69.9 (1998): 1062-1067.

9. Fee L. "Socket preservation". British Dental Journal 222.8 (2017).

10. Misch CE and Dietsh F. "Bone-grafting materials in implant dentistry”. Implant Dentistry 2.3 (1993): 158-167.

11. Anil S., et al. "Dental Implant Surface Enhancement and Osseointegration". In: Implant Dentistry - A Rapidly Evolving Practice. InTech (2011). doi:10.5772/16475

12. Porrini R., et al. "Alveolar bone regeneration in post-extraction socket: A review of materials to postpone dental implant". Biomedical Materials and Engineering 21.2 (2011): 63-74.

13. Juliana Dreyer MENEZES., et al. "Bioactive glass added to autogenous bone graft in maxillary sinus augmentation: a prospective histomorphometric, immunohistochemical, and bone graft resorption assessment". Journal of Applied Oral Science 26 (2018).

14. Waasdorp J and Reynolds MA. "Allogeneic bone onlay grafts for alveolar ridge augmentation: a systematic review". International Journal of Oral and Maxillofacial Implants 25 (2010): 525-531.

15. Rodella LF., et al. "Biomaterials in maxillofacial surgery: membranes and grafts". International Journal of Biomedical Science 7.2 (2011): 81-88.

16. Intini G. "The use of platelet-rich plasma in bone reconstruction therapy". Biomaterials 30.28 (2009): 4956-4966.

17. Marx RE., et al. "Platelet-rich plasma: Growth factor enhancement for bone grafts". Oral Surgery, Oral Medicine, Oral Pathology, and Oral Radiology 85.6 (1998): 638-646.

18. Dohan Ehrenfest DM., et al. "Classification of Platelet Concentrates: From Pure Platelet-Rich Plasma (P-PRP) to Leucocyteand Platelet-Rich Fibrin (L-PRF)". 7 (2009). doi:10.1016/j. tibtech.2008.11.009
19. Naik B., et al. "Role of Platelet rich fibrin in wound healing: A critical review". Journal of Conservative Dentistry 16.4 (2013): 284-293.

20. Dong-Seok Sohn BH., et al. "Utilization of Autologous Concentrated Growth Factors (CGF) Enriched Bone Graft Matrix (Sticky Bone) and CGF-Enriched Fibrin Membrane in Implant Dentistry". Journal of Implant and Advanced Clinical Dentistry 7.10 (2015): 17-29.

21. Dahlin C., et al. "Healing of bone defects by guided tissue regeneration". Plastic and Reconstructive Surgery 815 (1998): 672-676.

22. Wang H-L and Boyapati L. "\&quot;PASS\&quot; principles for predictable bone regeneration". Implant Dentistry 15.1 (2006): 8-17.

23. Wang H-L and Al-Shammari K. "HVC ridge deficiency classification: a therapeutically oriented classification". International Journal of Periodontics and Restorative Dentistry 22.4 (2002): 335-343.

24. Goodarzi Pour D., et al. "Accuracy of Cone Beam Computed Tomography for Detection of Bone Loss". Journal of Dentistry (Tehran). 12.7 (2015): 513-523.

25. Sartori S., et al. "Ten-year follow-up in a maxillary sinus augmentation using anorganic bovine bone (Bio-Oss). A case report with histomorphometric evaluation". Clinical Oral Implants Research 14.3 (2003): 369-372.

26. Barone A., et al. "Extraction Socket Healing in Humans After Ridge Preservation Techniques: Comparison Between Flapless and Flapped Procedures in a Randomized Clinical Trial". Journal of Periodontology 85.1 (2014): 14-23.

27. Jensen SS., et al. "Bone healing and graft resorption of autograft, anorganic bovine bone and $\beta$-tricalcium phosphate. A histologic and histomorphometric study in the mandibles of minipigs". Clinical Oral Implants Research 17.3 (2006): 237243.

28. Iasella JM., et al. "Ridge Preservation with Freeze-Dried Bone Allograft and a Collagen Membrane Compared to Extraction Alone for Implant Site Development: A Clinical and Histologic Study in Humans". Journal of Periodontology 74.7 (2003): 990999. 
Alveolar Ridge Preservation in Mandibular Molars Using Mixture of Anorganic Bovine Bone (ABB) AND Autogenous Particulate vs Mixture of Injectable Platelets Rich Fibrin, ABB and Autogenous Particulates (Sticky Bone) (Randomized Clinical Trial)

29. Van Der Weijden F., et al. "Alveolar bone dimensional changes of post-extraction sockets in humans: A systematic review". Journal of Clinical Periodontology 36.12 (2009): 1048-1058.

30. Tatullo M., et al. "Platelet rich fibrin (P.R.F.) in reconstructive surgery of atrophied maxillary bones: Clinical and histological evaluations". International Journal of Medical Sciences 9.10 (2012): 872-880.

31. Scarano A., et al. "Three-dimensional architecture and mechanical properties of bovine bone mixed with autologous platelet liquid, blood, or physiological water: An in vitro study". International Journal of Malecular Sciences 19.4 (2018).

32. Scarano A., et al. "Maxillary sinus augmentation with different biomaterials: a comparative histologic and histomorphometric study in man". Implant Dentistry 15.2 (2006): 197-207.

33. Zaffe D., et al. "Histological study on sinus lift grafting by Fisiograft and Bio-Oss". Journal of Materials Science: Materials in Medicine 16.9 (2005): 789-793.

34. Traini T., et al. "A Histologic and Histomorphometric Evaluation of Anorganic Bovine Bone Retrieved 9 Years After a Sinus Augmentation Procedure". Journal of Periodontology 78.5 (2005): 955-961.

35. Guarnieri R., et al. "Histologic and histomorphometric analysis of bone regeneration with bovine grafting material after 24 months of healing. A case report". Journal of Functional Biomaterials 9.3 (2018).

36. Piattelli M., et al. "Bone reactions to anorganic bovine bone (Bio-Oss) used in sinus augmentation procedures: a histologic long-term report of 20 cases in humans". International Journal of Oral and Maxillofacial Implants 14.6 (2019): 835-840.

37. Fujioka-Kobayashi M., et al. "Optimized Platelet-Rich Fibrin With the Low-Speed Concept: Growth Factor Release, Biocompatibility, and Cellular Response". Journal of Periodontology 88.1 (2017): 112-121.

38. Miron RJ., et al. "Injectable platelet rich fibrin (i-PRF): opportunities in regenerative dentistry?" Clinical Oral Investigation 21.8 (2017): 2619-2627.
39. Daugela P., et al. "Influence of leukocyte- and platelet-rich fibrin (L-PRF) on the outcomes of impacted mandibular third molar removal surgery: A split-mouth randomized clinical trial". Quintessence International 49.5 (2018): 377-388.

\section{Assets from publication with us}

- Prompt Acknowledgement after receiving the article

- Thorough Double blinded peer review

- Rapid Publication

- Issue of Publication Certificate

- High visibility of your Published work

Website: https://www.actascientific.com/

Submit Article: https://www.actascientific.com/submission.php Email us: editor@actascientific.com

Contact us: +919182824667 